\title{
Development of a Numerical Model for Vibration Analysis of Low Folded Shells under Dynamic Actions
}

\author{
Georges Adjibola A. Ale ${ }^{1}$, Emmanuel E. T. Olodo ${ }^{1}$, Valery Doko ${ }^{1} \&$ Antoine Vianou $^{2}$ \\ ${ }^{1}$ Laboratory of Energetic and Applied Mechanics (LEMA), University of Abomey-Calavi, Benin \\ ${ }^{2}$ Polytechnic School of Abomey-Calavi, University of Abomey-Calavi, Benin \\ Correspondence : Emmanuel E. T. Olodo. Laboratory of Energetic and Applied Mechanics (LEMA)/University \\ of Abomey-Calavi, 01 BP 2009 Cotonou, Bénin.
}

Received: March 7, 2020

Accepted: March 29, 2020

Online Published: March 30, 2020

doi:10.5539/mas.v14n4p111

URL: https://doi.org/10.5539/mas.v14n4p111

\begin{abstract}
In this work a numerical model is developed for vibration analysis of low folded shells under dynamic actions. At first it is done to describe the used finite element discrete model based on Lagrange variational principles. To solve the eigen value problem of these structures a numerical algorithm is proposed using Householder's QR-iteration transformations. This method provides a tridiagonal matrix whose eigen values coincide with those of the initial matrix and significantly reduces the iteration number compared to the Lanczos method. Implementation of the method is carried out on seven folded shell mathematical models. Obtained results show that accuracy can be improved and computational time can be significantly reduced compared to the methods available in the technical literature for this class of problems.
\end{abstract}

Keywords: folded shells, numerical model, eigenvalues

\section{Introduction}

Several finite element models are proposed for structure modeling. Thus for thin structures we have elements such as shell, plate, etc. However, when faced with structures with very contrasting geometries like folded shells one of the main difficulties is the structure discreting allowing a better accuracy of the expected results. Among the first to attempt to provide an answer to the shell problem was (Ahmad et al.1970) in the 1970s. These models, called degenerate three-dimensional finite elements are based on volume-based iso-parametric elements with only two nodes following thickness direction. The advantage of this modeling lies in the exclusive use of freedom degrees in displacement. However it suffers from change in the principle of virtual powers which requires a changé in the material's behavior law. Several authors such as (Parisch et al.,1979), (Brendel et al.,1980 ; Ramm et al., 1980), (Kim et al., 2005) nevertheless valued this approach.

For the case of folded shells one of the main problems is related to compliance difficulties. Indeed in folded structures during shell junctions along the edges non-compliance becomes almost inevitable. If we consider for example two finite elements connected by right angles it is immediately observed that each element has a rotational freedom degree which cannot be connected to the other element since there are only two rotational freedom degrees per node. This circumstance, a natural consequence of modeling can lead to continuity problems.

Solving of vibration problem consist in evaluation of damping properties wich are resonance pulsation and modal damping. Several numerical approaches are available in the technical literature including the Lanczos method (Lanczos, 1950), Arnoldi's method, (Arnoldi, 1951), the QR method (Bate, 1982) or the subspace iterative method (Leung, 1995). One can cite the iteration-reduction method (Chen et al.,1999) which involves solving the eigenvalue problem in two steps. The iteration-reduction method requires much iterations, which significantly increases the computational time for large structures. An alternative based on the disturbance and homotopy technique is therefore developed by Daya and Potier-Ferry (Daya et al., 2001). Other finite element models for shell vibration analysis have been developed by (Patel et al., 2000 ; Asokendu et al., 2004 ; Damatty et al., 2005 ; Nguyen-Thoi et al., 2018 ; Pathok et al., 2019).

In this work a shell finite element model is proposed in which Householder's QR transformations are used to solve the eigenvalue problem of folded shells. Because this method allows to obtain a tri-diagonal matrix whose 
eigenvalues coincide with those of the initial matrix.and significantly reduces the iteration number compared to the Lanczos method.

The main advantage of the proposed method is that it improves accuracy and significantly reduces computational time compared to the methods available in the technical literature for this class of problems.

This work is structured in four sections. Introduction : in this section a bibliographic synthesis of proposed works for the numerical analysis of eigenvalue problems is carried out. It outlines the advantages and inconvenients of one compared to the other and a new approach is proposed to improve accuracy and computational time compared to the methods available in the literature. Methodology: in this section is carried out a finite element formulation of the problem in dynamics of low shells and a method of solving the eigenvalue problem by Householder's transformations is proposed. Results and discussion: This section exposes and analyzes the results obtained as a result of the implementation of the method on different types of shells. The conclusion summarizes the main results and offers prospects for the future development of the work proposed here. A list of bibliography is drawn up at the end of the document.

\section{Material and methods}

\subsection{Finite Element Formulation of the Problem in Low Shell Dynamics}

\subsubsection{Description of the discrete model of used finite elements}

For construction of the mathematical model in free and forced vibrations of the shell element will be modeled with the use of second-order iso-parametric curvilinear quadrangle elements at eight nodes with variable characteristics (Figure 1).

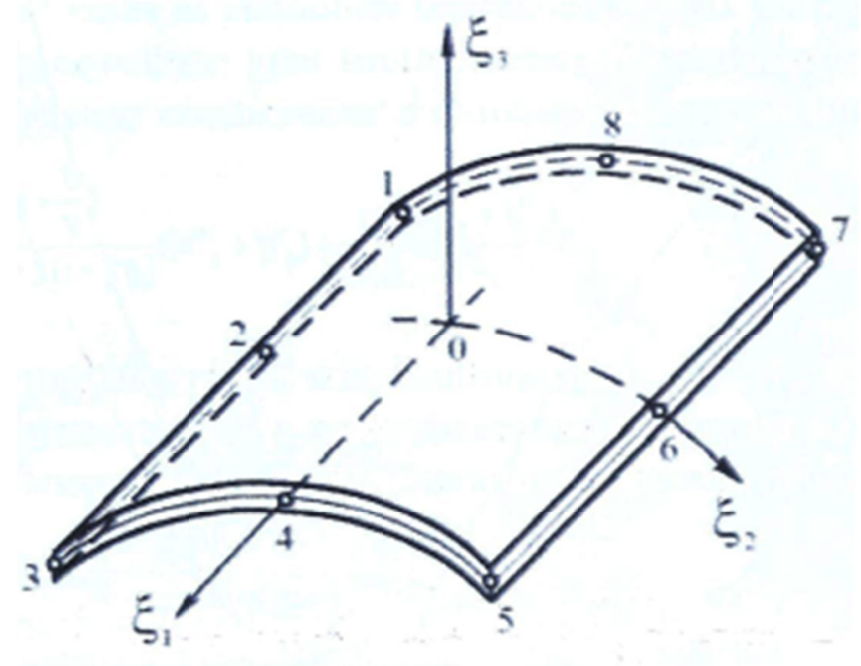

Figure 1. Eight-node iso-parametric curvilinear element

Each node is associated with six freedom degrees: three displacements, two rotations and one torsion. Each finite element is associated with a local coordinate system $\left(\xi_{1}, \xi_{2}, \xi_{3}\right)$ and different displacement approximation orders according to normal on medium plane (quadratic law) and following the tangent to the shell middle plane. This allows to describe with sufficient accuracy geometry of the elements and the displacement distribution. The finite element sides correspond to the coordinates $\left(\xi_{1}, \xi_{2}\right)$. The main advantage of this element over other elements is that it ensures convergence to the actual solution for a densified mesh.

Either $u_{1}, u_{2}, u_{3}$ the components of the vector $\vec{u}$ - node displacements. Projections of nodal displacement vector in global coordinates can be expressed as follows (Zienkiewicz, 2000) :

$$
\mathrm{u}_{\mathrm{k}}=\sum_{\mathrm{i}=1}^{8} u_{k}^{(\mathrm{i})}{ }^{(\mathrm{i})} \mathrm{N}_{\mathrm{i}}\left(\xi_{1}, \xi_{2}\right)(\mathrm{k}=\overline{1,3}) \text {, }
$$

$u_{k}^{(i)}$ - projections of displacement vector at the $i$-node,

$N_{i}\left(\xi_{1}, \xi_{2}\right)$ - the shape functions of element in the local coordinates.

Displacements at the elementary level can be determined by taking into account (2.1) in following form: 


$$
\left\{\begin{array}{l}
u_{1} \\
u_{2} \\
u_{3}
\end{array}\right\}=\sum_{i=1}^{8} \quad \mathrm{Ni}\left(\begin{array}{llll}
\xi & 1, & \xi & 2
\end{array}\right)\left\{\begin{array}{l}
u_{1}^{(i)} \\
u_{2}^{(i)} \\
u_{3}^{(i)}
\end{array}\right\}+\sum_{i=1}^{8} \operatorname{Ni}\left(\begin{array}{llll}
\xi & 1, & \xi & 2
\end{array}\right) \cdot \xi 3 \cdot \frac{h i}{2}\left(\left\{\begin{array}{c}
v_{1}^{1 i} \\
v_{2}^{1 i} \\
v_{3}^{1 i}
\end{array}\right\} \theta_{1}^{(i)}-\left\{\begin{array}{l}
v_{1}^{2 i} \\
v_{2}^{2 i} \\
v_{3}^{2 i}
\end{array}\right\} \theta_{2}^{(i)}\right)
$$

Here, $\left\{u^{(i)}\right\}=\left\{u_{1}^{(i)}, u_{2}^{(i)}, u_{3}^{(i)}\right\}-$ the nodal displacement vector,

$h_{i}$ - element thickness at i-node,

$\bar{v}_{1}=\left\{v_{1}^{1 i}, v_{2}^{1 i}, v_{3}^{1 i}\right\}, \bar{v}_{2}=\left\{v_{1}^{2 i}, v_{2}^{2 i}, v_{3}^{2 i}\right\}$ - orthonormed vectors,

$\theta_{1}^{(i)}, \theta_{2}^{(i)}$ - normal to the i-node rotational angles in relation to the vectors $\bar{v}_{1}$ and $\bar{v}_{2}$ assuming a summons of repeated indexes.

The element shape functions are given by :

$$
\begin{gathered}
\mathrm{N} 1=-\frac{1}{4}\left(1-\xi_{1}\right)\left(1-\xi_{2}\right)\left(\xi_{1}+\xi_{2}+1\right) . \mathrm{N} 2=\frac{1}{2}\left(1-\xi_{1}^{2}\right)(1-\xi 2), \\
\mathrm{N} 3=\frac{1}{4}\left(1+\xi_{1}\right)\left(1-\xi_{2}\right)\left(\xi_{1}-\xi_{2}-1\right) \mathrm{N} 4=\frac{1}{2}\left(1+\xi_{1}\right)\left(1-\xi_{2}^{2}\right), \\
\mathrm{N} 5=\frac{1}{4}\left(1+\xi_{1}\right)\left(1+\xi_{2}\right)\left(\xi_{1}+\xi_{2}-1\right) \mathrm{N} 6=\frac{1}{2}\left(1-\xi_{1}^{2}\right)\left(1+\xi_{2}\right), \\
\mathrm{N} 7=-\frac{1}{4}\left(1-\xi_{1}\right)\left(1+\xi_{2}\right)\left(\xi_{1}-\xi_{2}+1\right), \mathrm{N} 8=\frac{1}{2}(1-\xi 2)\left(1-\xi_{2}^{2}\right) .
\end{gathered}
$$

At each node we have three displacements $u_{1}, u_{2}, u_{3}$, two rotations $\theta_{1}, \theta_{2}$ and a torsion $\theta_{12}$.

The generalized vector of nodal displacements is as follows:

$$
\left\{q^{(e)}\right\}=\left\{u_{1}^{1}, u_{2}^{1}, u_{3}^{1}, \theta_{1}^{1}, \theta_{2}^{1}, \theta_{12}^{1}, \ldots, u_{1}^{8}, u_{2}^{8}, u_{3}^{8}, \theta_{1}^{8}, \theta_{2}^{8}, \theta_{12}^{8}\right\}^{T} .
$$

To determine the Jacobean matrix we will establish relationship between shape function derivatives of the element following global coordinate axes $\left(\propto_{1}, \propto_{2}, \xi\right)$ and their derivatives following the local axes $\left(\xi_{1}, \xi_{2}, \xi_{3}\right)$ :

$$
\begin{gathered}
{\left[\frac{\partial N}{\partial \alpha_{1}} \frac{\partial N}{\partial \alpha_{2}} \frac{\partial N}{\partial \xi}\right]=[J]^{-1}\left[\frac{\partial N}{\partial \xi_{1}} \frac{\partial N}{\partial \xi_{2}} \frac{\partial N}{\partial \xi_{3}}\right],} \\
{[J]=\left[\begin{array}{lll}
\partial \alpha_{1} / \partial \xi_{1} & \partial \alpha_{2} / \partial \xi_{1} & \partial \xi / \partial \xi_{1} \\
\partial \alpha_{1} / \partial \xi_{2} & \partial \alpha_{2} / \partial \xi_{2} & \partial \xi / \partial \xi_{2} \\
\partial \alpha_{1} / \partial \xi_{3} & \partial \alpha_{2} / \partial \xi_{3} & \partial \xi / \partial \xi_{3}
\end{array}\right],}
\end{gathered}
$$

\subsubsection{Finite element free vibration formulation of low shells}

For the free vibration problem of low thin shells we will start from Lagrange variational principle (Zienkiewich, 2000):

$$
\frac{d}{d t}\left(\frac{\partial \ni}{\partial \dot{q}}\right)-\frac{\partial \ni}{\partial q}=o,
$$

Here, $\exists=\Pi-T$ is Lagrange's function,

$\Pi, T$ - the potential and kinetic energies of deformation and vibration of the discretic shell,

q- the nodal displacement vector of the finite element model.

The potential deformation energy of the system in matrix form will be:

$$
\Pi=\frac{1}{2} \iiint_{v}\{q\}^{T} B^{T} D_{\sigma}\{q\} d v=\frac{1}{2}\left(\{q\}^{T} K\{q\}\right),
$$

B - matrix of gradients of the finite element,

$D_{\sigma}$ - the elasticity matrix.

Hence the rigidity matrix $\mathrm{K}$ of the shell finite element model will be:

$$
\mathrm{K}=\iiint_{V} B^{T} D_{\sigma} B \mathrm{dV} \text {. }
$$

Vibration kinetic energy of the system's will be determined as the sum of the elemental energies:

$$
\mathrm{T}=\frac{1}{2} \rho \iiint_{V}\{q\}^{T} N^{T} N\{q\} \mathrm{dV}=\frac{1}{2}\left(\{q\}^{T} M\{q\}\right) .
$$


The mass matrix of the system will be deduced from (2.10) by the following expression :

$$
\mathrm{M}=\rho \iiint_{V} N^{T} N \mathrm{dV} .
$$

The matrix writing of shell free vibration equations without damping will be:

$$
\mathrm{M}\{\ddot{q}\}+\mathrm{K}\{\mathrm{q}\}=\{0\},
$$

$\{q\},\{\mathrm{q}\}$ - the nodal acceleration vector and nodal displacement vector.

The elementary rigidity and mass matrices on element of $V_{e}$ volume will be determined by the following expressions :

$$
K^{(e)}=\iiint_{V_{e}} B^{(e)^{T}} D_{\sigma} B^{(e)} d V_{e}, M^{(e)}=\rho \iiint_{V_{e}} N^{T} N d V_{e} .
$$

Deformation vector of the shell finite element will be written:

$$
\left\{\varepsilon^{(e)}\right\}=B^{(e)}\left\{q^{(e)}\right\},
$$

$B(e)$ - shape function gradient matrix on the element

$q(e)$ - nodal displacemen vector on the element.

We will look for solution of linear system (2.12) in following form:

$$
\{q\}=\left\{q_{0}\right\} \cos \left(\omega_{i} t+\varphi_{i}\right),
$$

Qoi- eigen vector associated with the $i^{\text {th }}$-eigen mode,

$\omega_{i}-i^{\text {th }}$ - eigenpulse.

Taking into account relationship (2.15) equation (2.12) becomes:

$$
\left(-\omega_{i}^{2} \mathrm{M}+\mathrm{K}\right)\left\{q_{o}\right\}_{i}=\{0\}
$$

As $\left\{q_{o}\right\}_{i} \neq\{0\}$, the system's $(2.16)$ non-trivial solution requires:

$$
\operatorname{det}\left(-\omega_{i}^{2} \mathrm{M}+\mathrm{K}\right)=0 \text {. }
$$

Due to the orthogolinal conditions of the eigenvectors associated with the matrix $\mathrm{M}$ we have :

$$
\left\{q_{0}\right\}_{i}^{T} \mathrm{M}\left\{q_{0}\right\}_{j}=\left\{\begin{array}{l}
0 ; i \neq j \\
1 ; i=j
\end{array} .\right.
$$

So equation (2.16) taking into account (2.18) becomes eigenvalue problem that can be put in the following form :

$$
\Lambda\{x\}-\lambda \phi\{x\}=\{0\},
$$

$\Lambda$ and $\phi$ - positive defined symmetrical matrices,

$\lambda$ - the eigenvalues,

$\{x\}$ - the eigen vectors.

\subsection{Householder Algorithm Solving}

To solve the problem (2.16) we will use the Householder-QR algorithm with reverse integration which compared to the Lanczos method significantly reduces the computational time. Householder's algorithm can be used for a class of standard eigenvalue problems with following form :

$$
\Lambda\{x\}-\lambda I\{x\}=\{0\},
$$

$I$ - unit matrix

For solving of generalized eigenvalue problem by Householder-QR algorithm equation (2.16) refers to the standard form (2.20). This transformation can be done using Cholesky's decomposition for the matrix M (Bate et al., 1982). In this case we get:

$$
\Lambda=L^{-1} \mathrm{~K}\left(L^{T}\right)^{-1},
$$

here $L\left(L^{T}\right)=\mathrm{M}$; ( $L$ being the lower triangular matrix with positive diagonal in Cholesky's decomposition).

Cholesky's decomposition exists if matrix M is symmetrical and defined as positive. For our case this condition is satisfied. The elements of matrix $\mathrm{L}$ are calculated starting with the upper left angle of matrix $\mathrm{M}$ by the following expressions : 


$$
L_{i i}=\sqrt{M_{i i}-\sum_{k=1}^{i-1} L^{2}{ }_{i k}}(\mathrm{i}=1, \ldots, \Theta),
$$

$\Theta$ - System freedom degree.

2.2.1 The main steps in Householder-QR algorithm implementing

1. Tridiagonalization of the initial matrix $\Lambda$ using Householder transformation taking into account (2.22).

2. Computing all the eigenvalues using $Q R$ transformations ;

3. Computing the eigen vectors of tridiagonal matrix's using reverse iterations.

Algorithm's step description

The tridiagonalization stage of the matrix $\Lambda$ consists of following $(\Theta-2)$ iterations (Wilkinson, 1970) :

$$
\Lambda_{r}=P_{r} \Lambda_{r-1} P_{r},(\mathrm{r}=1 \ldots, \Theta-2)
$$

P- Householder matrix calculated by the following formula (Wilkinson,1970):

$$
\begin{gathered}
P_{r}=I \frac{\{V\}_{r}\{V\}_{r}^{T}}{2 D_{r}^{2}}, \\
V_{r, i}=0(i=1,2, \ldots, r), V_{r, r+1}=\Lambda_{r, r+1} \mp S_{r,} \\
V_{r, i}=\Lambda_{r, i(i=r+2, \ldots, \Theta),} \\
S_{r}=\left(\sum_{i=r+1}^{\Theta} \Lambda_{r, i}^{2}\right)^{1 / 2}, \\
2 D_{r}^{2}=S_{r}^{2} \mp \Lambda_{r, r+1} S_{r}
\end{gathered}
$$

At initial stage we adopt $\Lambda_{0}=\Lambda$. When algorithm is executed transformation (2.23) cancels out at the r-line and $r$ column. The same is true for all the elements lower to the right of the tridiagonal matrix.

In relationships (2.25) $S_{r}$ is the same sign as element $\Lambda_{r, r-1}$. The formulas are written in such a way that only the elements of the upper triangle of the matrix $\Lambda_{r-1}$ are retained. The current element of the matrix $\Lambda_{r-1}$, (with the $\mathrm{i}, \mathrm{j}$ index for example) will be noted $\Lambda_{i, j}$. The current element of the vector $V_{r}$ (with $i$-index for example) will be noted $V_{r, i}$. The $P_{r}$ matrices are orthogonal and symmetrical.

Expression (2.24) can be written in following form:

$$
P_{r}=\mathrm{I}-2\{W\}_{r}\{W\}_{r}^{T},
$$

here $\{W\}_{r}=\frac{\{V\}_{r}}{2 D_{r}},\{W\}_{r}^{T}\{W\}_{r}=1$.

Householder method allows for the tridiagonal symmetrical matrix whose eigenvalues coincide with those of the original matrix. Advantage of this method is the significant reduction in the calculation number compared to other methods.

The next step is to calculate all the eigenvalues of the tridiagonal matrix $\Lambda$ obtained using QR transformations. The tridiagonal matrix $\Lambda$ is written according to Graham-Schmidt's decomposition into a product of Q.R, Q being an orthogonal matrix $\left(Q^{T} Q=I\right)$ and R- the upper triangular matrix.

For calculation of R and Q matrices one use Householder's transformations with the symmetrical and orthogonal $P_{r}$ matrices taking into account (2.26). This guarantees the orthogoality of matrix $\mathrm{Q}$.

Matrix $\mathrm{Q}$ is calculated by formula $Q=P_{1} \cdot P_{2} \cdot P_{3} \ldots . P_{\theta-1}$, keeps itself as comultiplier series and is not calculated in explicit form. Matrix $\mathrm{R}$ is result of a succession of operations:

$$
P_{1} \Lambda, P_{2} \mathrm{P} 1 \Lambda, \ldots, P_{\Theta-1} P_{\Theta-2} \ldots P_{1} \Lambda .
$$

At the next iteration the matrix $\Lambda$ is obtained as the product of the previous iteration by Q left and right.

This QR-iterations algorithm is given by the following relationships (Strang,1980) :

$$
\begin{aligned}
\Lambda_{0} & =\Lambda, \\
\Lambda_{i} & =Q_{i} R_{i} \\
\Lambda_{i+1} & =Q_{i}^{T} \Lambda_{i} Q_{i}=Q_{i}^{T} Q_{i} R_{i} Q_{i}=R_{i} Q_{i}
\end{aligned}
$$

Relationships (2.27) and (2.28) describe algorithms without deviations. However, to accelerate the convergence of the QR algorithm one use deviations $\alpha_{i}$ (values close to eigenvalues). In this case the expressions (2.28) are as follows:

$$
\Lambda_{0}=\Lambda
$$




$$
\begin{aligned}
& \Lambda_{i}-\alpha_{i} I=Q_{i} R_{i,} \\
& \Lambda_{i+1}=R_{i} Q_{i}+\alpha_{i} I
\end{aligned}
$$

If as gap $\alpha_{i}$ one takes a very different value from the sought eigenvalue the solution can be erroneous. For this in practice as deviation $\alpha_{i}$ from the $i^{\text {th }}$-iteration it is more convenient to choose the lower right element of the matrix $\Lambda$.

The tridiagonal matrix eigen vectors obtained by Householder method are calculated using reverse iterations by following formula (Bate,1982) :

$$
\left(\Lambda-\lambda_{i} I\right)\left\{\Psi_{i}\right\}^{(k+1)}=\left\{\Psi_{i}\right\}^{(k)}(\mathrm{k}=1,2 \mathrm{I}=1,2, \ldots, \Theta)
$$

$\left\{\psi_{i}\right\}-i^{\text {th }}$ vector of the tridiagonal matrix $\Lambda$

$\mathrm{k}$ - iteration number.

The tridiagonal matrix's eigen vectors are transformed using previously obtained Householder matrixes (2.26) into eigen vectors of the original standard problem (Bate 1982) :

$$
\left\{x_{i}\right\}=P_{1} P_{2} \quad \ldots P_{\Theta-2} \quad\left\{\Psi_{i}\right\}(\mathrm{i}=1, \ldots, \Theta),
$$

$\left\{x_{i}\right\}-i^{\text {th }}$ eigen vector of the original problem;

$$
P_{r} \text { - Householder matrix }(r=1 \ldots . \theta-2) .
$$

After determining the initial problem eigen vectors by formula (2.31), they become eigen vectors of the expanded problem following relationship (2.21). As a result, the elements of the matrix $\Lambda$ in the columns are replaced by the elements of calculated vectors $\left\{q_{0}\right\}_{i}$ that determine the eigen forms of vibrations.

By the calculated eigen vectors $\left\{q_{0}\right\}_{i}$ using expression (2.15), we determine the angular displacement vector of the structure for each frequency $f_{i}$.

\section{Results and Discussion}

Eight shell models are considered:

- four $8 \times 8 \mathrm{~m}$ square-plan, $12 \times 12 \mathrm{~m}$ square-plan low-plan shells (figure 2);

- four low folded shells with rectangular plane $8 \times 12 \mathrm{~m}, 8 \times 16 \mathrm{~m}$ and $8 \times 20 \mathrm{~m}$ (figure 3).

Figures $2(\mathrm{a}, \mathrm{b}, \mathrm{c}, \mathrm{d})$ show geometric models of low shells on square plane corresponding respectively to parameters $k=l=0 ; k=l=1 ; k=l=2$ and $k=l=3$.

Figures 3 (a, b, c, d) show geometric models of low folded shells on rectangular plane corresponding respectively to parameters $k=2, l=2 ; k=2, l=3 ; k=2, l=4$ and $k=2, l=5$. 
a
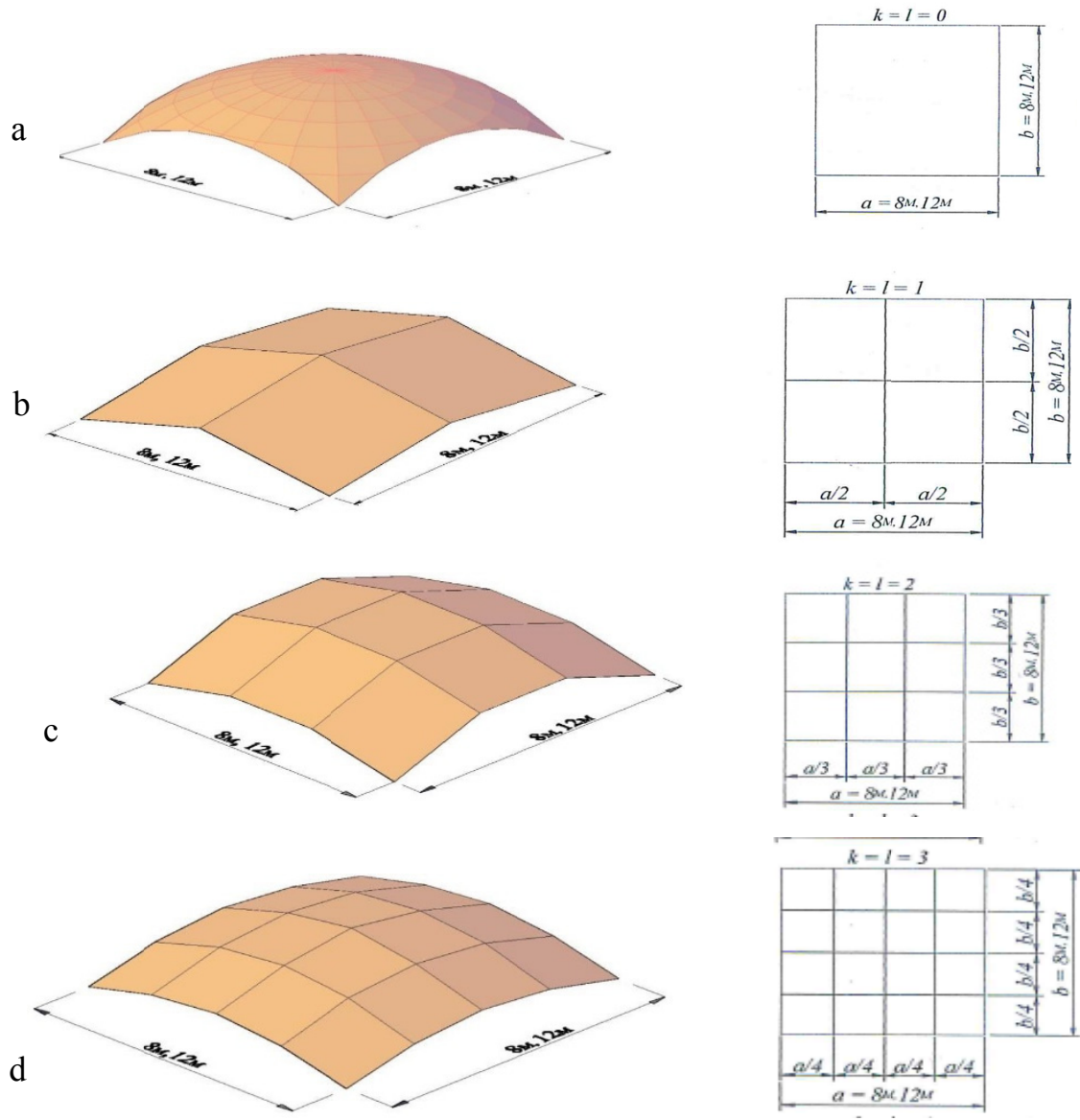

Figure 2. Mathematical models of square-plan low shells 

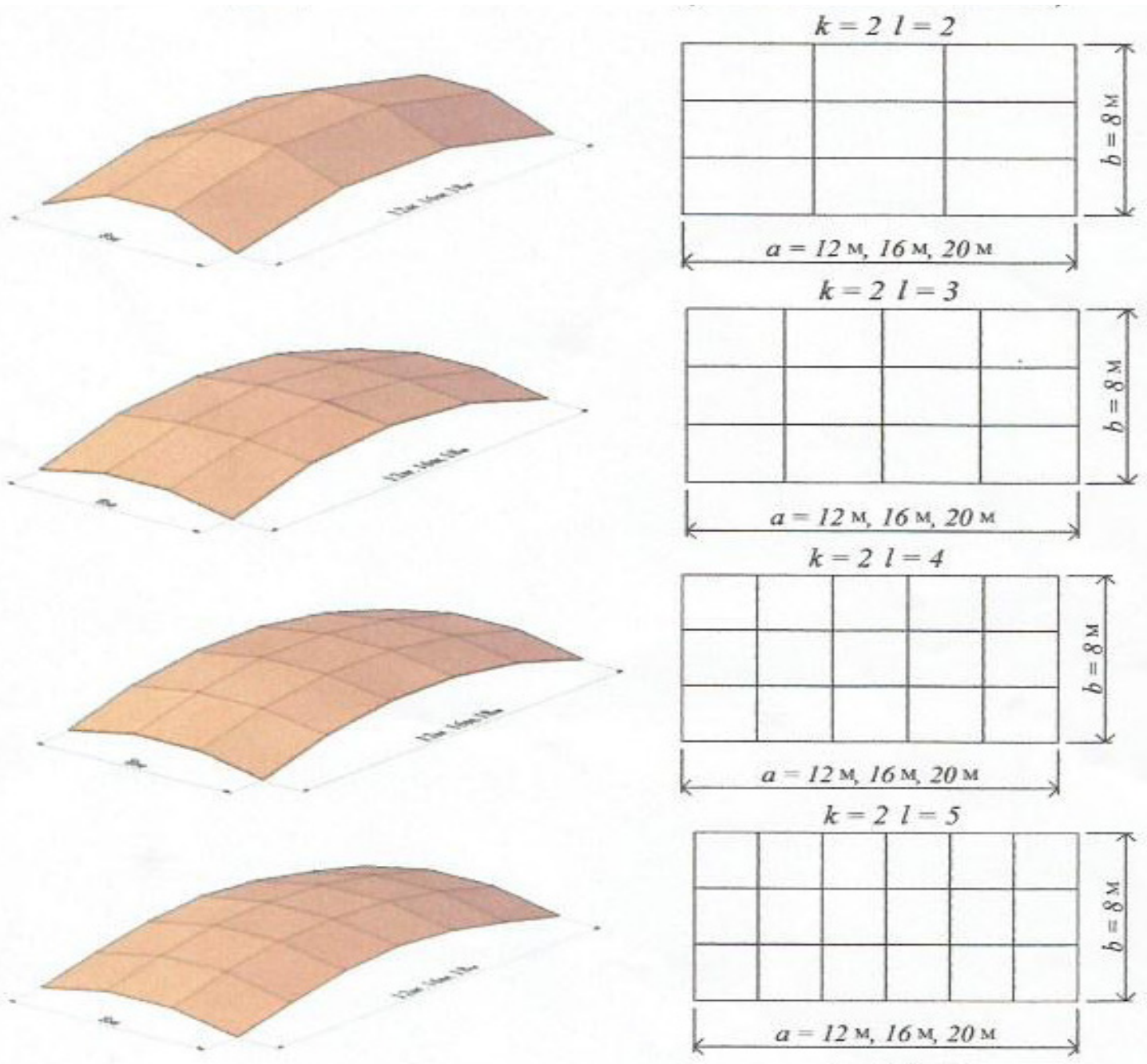

Figure 3. Mathematical models of low rectangular-plan folded shells

The results of the calculation of the free vibration frequencies of square-plan folded shells are shown in tables $1-4$.

Table 1. Free vibration frequency values of the $8 \times 8 \mathrm{~m}$ square shell

\begin{tabular}{cllllllllllll}
\hline \multicolumn{10}{c}{ Shell $8 \times 8 M$} \\
\hline$m=n$ & 1 & 2 & 3 & 4 & 5 & 6 & 7 & 8 & 9 & 10 & 11 & 12 \\
\hline$k=l=0$ & 63.9 & 65.2 & 70.4 & 82.8 & 104.2 & 134.7 & 173.7 & 220.4 & 274.5 & 335.8 & 403.9 & 478.9 \\
$k=l=1$ & 46.2 & 13.2 & 54.8 & 52.7 & 94.4 & 118.7 & 168.0 & 211.0 & 271.0 & 329.6 & 401.5 & 474.7 \\
$k=l=2$ & 51.2 & 52.8 & 29.7 & 73.4 & 97.0 & 118.7 & 169.4 & 217.1 & 267.0 & 333.6 & 402.1 & 474.7 \\
$k=l=3$ & 44.2 & 46.0 & 53.2 & 52.7 & 93.5 & 126.6 & 167.4 & 211.0 & 270.6 & 332.6 & 401.3 & 474.7 \\
$k=l=4$ & 55.2 & 56.7 & 62.6 & 76.3 & 82.4 & 130.8 & 170.7 & 218.0 & 272.6 & 329.6 & 402.6 & 477.9 \\
$k=l=5$ & 54.2 & 55.7 & 61.7 & 75.6 & 98.6 & 118.7 & 170.3 & 217.8 & 272.4 & 334.0 & 402.5 & 474.7 \\
\hline
\end{tabular}


Table 2. Free vibration frequency values of the $12 \times 12 \mathrm{~m}$ square shell

\begin{tabular}{ccccccccccccc}
\hline \multicolumn{10}{c}{ Shell 12x12 $\mu$} \\
\hline $\mathrm{m}=\mathrm{n}$ & 1 & 2 & 3 & 4 & 5 & 6 & 7 & 8 & 9 & 10 & 11 & 12 \\
\hline$k=l=0$ & 46.0 & 46.3 & 47.8 & 51.6 & 58.8 & 69.9 & 85.2 & 104.4 & 127.3 & 153.5 & 183.1 & 215.9 \\
$k=l=1$ & 30.8 & 5.9 & 33.4 & 23.4 & 47.8 & 52.7 & 78.1 & 93.8 & 122.6 & 146.5 & 179.9 & 211.0 \\
$k=l=2$ & 34.1 & 34.6 & 13.2 & 41.4 & 50.0 & 52.7 & 79.5 & 99.8 & 118.7 & 150.4 & 180.5 & 211.0 \\
$k=l=3$ & 29.4 & 30.0 & 32.2 & 23.4 & 47.0 & 60.4 & 77.6 & 93.8 & 122.3 & 149.4 & 179.7 & 211.0 \\
$k=l=4$ & 36.8 & 37.2 & 39.0 & 43.6 & 36.6 & 64.3 & 80.6 & 100.7 & 124.2 & 146.5 & 181.0 & 214.1
\end{tabular}

Table 3. Free vibration frequency values of the $8 \times 8 \mathrm{~m}$ square shell for $\boldsymbol{k}=\boldsymbol{l}=\mathbf{2}$

\begin{tabular}{|c|c|c|c|c|c|c|c|c|c|c|c|c|}
\hline \multicolumn{13}{|c|}{ Shell $8 x 8$ M for $k=l=2$} \\
\hline$m=n$ & 1 & 2 & 3 & 4 & 5 & 6 & 7 & 8 & 9 & 10 & 11 & 12 \\
\hline$h=0.08 \mathrm{M}$ & 51.2 & 52.8 & 29.7 & 73.4 & 97.0 & 118.7 & 169.4 & 217.1 & 267.0 & 333.6 & 402.1 & 474.7 \\
\hline$h=0.09 \mathrm{M}$ & 51.3 & 53.2 & 33.4 & 78.3 & 105.9 & 133.5 & 188.8 & 242.8 & 300.4 & 374.3 & 451.6 & 534.0 \\
\hline$h=0.1 \mathrm{M}$ & 51.3 & 53.7 & 37.1 & 83.4 & 115.0 & 148.3 & 208.3 & 268.6 & 333.8 & 415.2 & 501.2 & 593.3 \\
\hline
\end{tabular}

Table 4. Free vibration frequency values of the $12 \times 12 \mathrm{~m}$ square shell for $\boldsymbol{k}=\boldsymbol{l}=\mathbf{2}$

Shell $12 \times 12$ ufor $k=l=2$

\begin{tabular}{lcccccccccccc}
\hline$m=n$ & 1 & 2 & 3 & 4 & 5 & 6 & 7 & 8 & 9 & 10 & 11 & 12 \\
\hline$h=0.08 \mathrm{м}$ & 49.3 & 50.1 & 23.7 & 62.6 & 75.1 & 84.4 & 139.6 & 171.4 & 209.6 & 252.6 & 304.7 & 356.3 \\
$h=0.09 \mathrm{M}$ & 49.8 & 50.9 & 25.8 & 68.5 & 84.7 & 95.5 & 158.3 & 193.2 & 230.2 & 290.4 & 352.8 & 410.3 \\
$h=0.1 \mathrm{M}$ & 50.1 & 51.3 & 27.4 & 71.3 & 94.8 & 118.1 & 168.6 & 216.3 & 266.7 & 331.8 & 401.2 & 473.8
\end{tabular}

Tables 1-4 show that the values of the free vibration frequencies of the studied square-plane low shells increase significantly when the wave number in both directions $m$ and $n$ is greater than or equal to 4 . On the other hand, when the shell dimensions on the plane are larger there is a decrease in frequency values. Tables 3 and 4 show that thickness has an influence on the free vibrations of the square-plan shell. Indeed, when we increase thickness, we observe an increase of the shell free vibration frequencies.

In figures 4-7 are shown the frequency variation of the square-plane low shells depending on the wave number $\mathrm{m}$ and $\mathrm{n}$ and the fold ridge number $\mathrm{k}$ and $\mathrm{l}$ in both directions. 


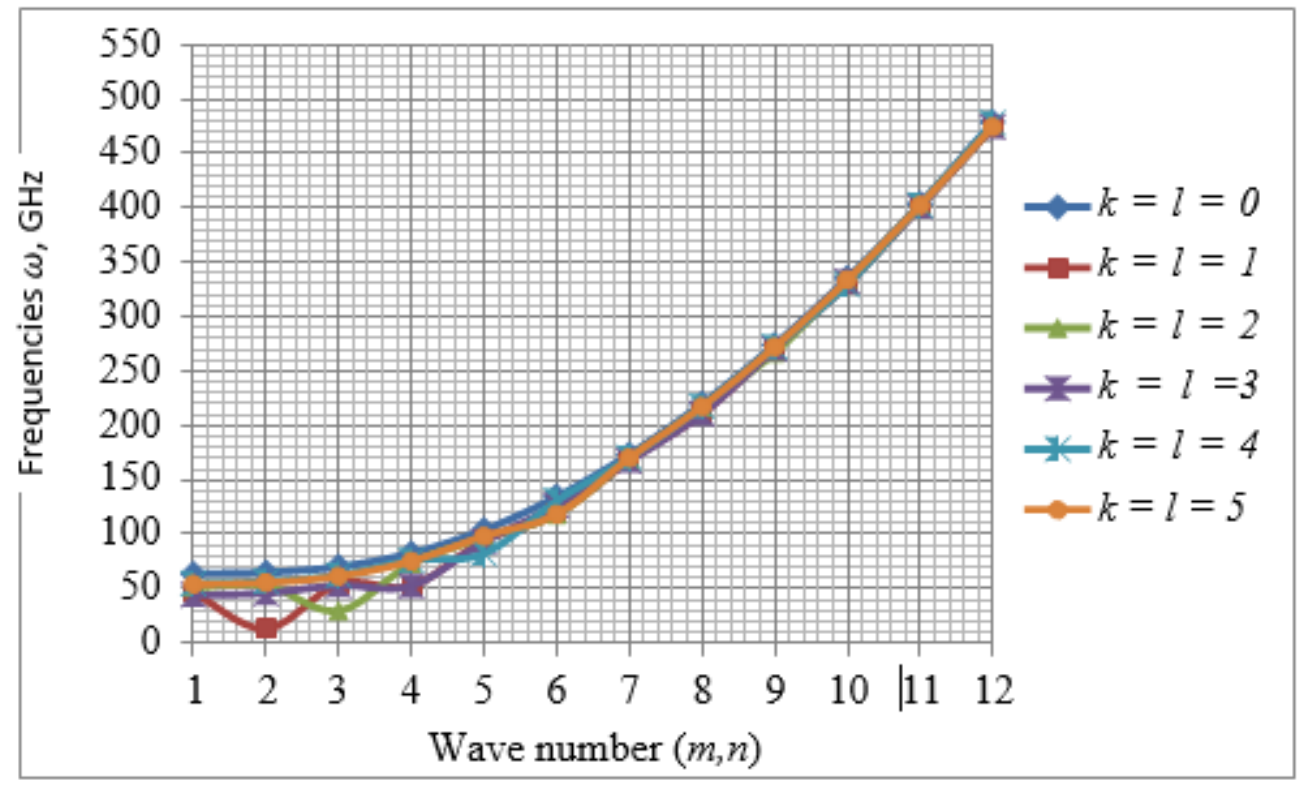

Figure 4. Frequency variation curves based on the wave numbers $(m, n)$ for $8 \times 8 m$ shell

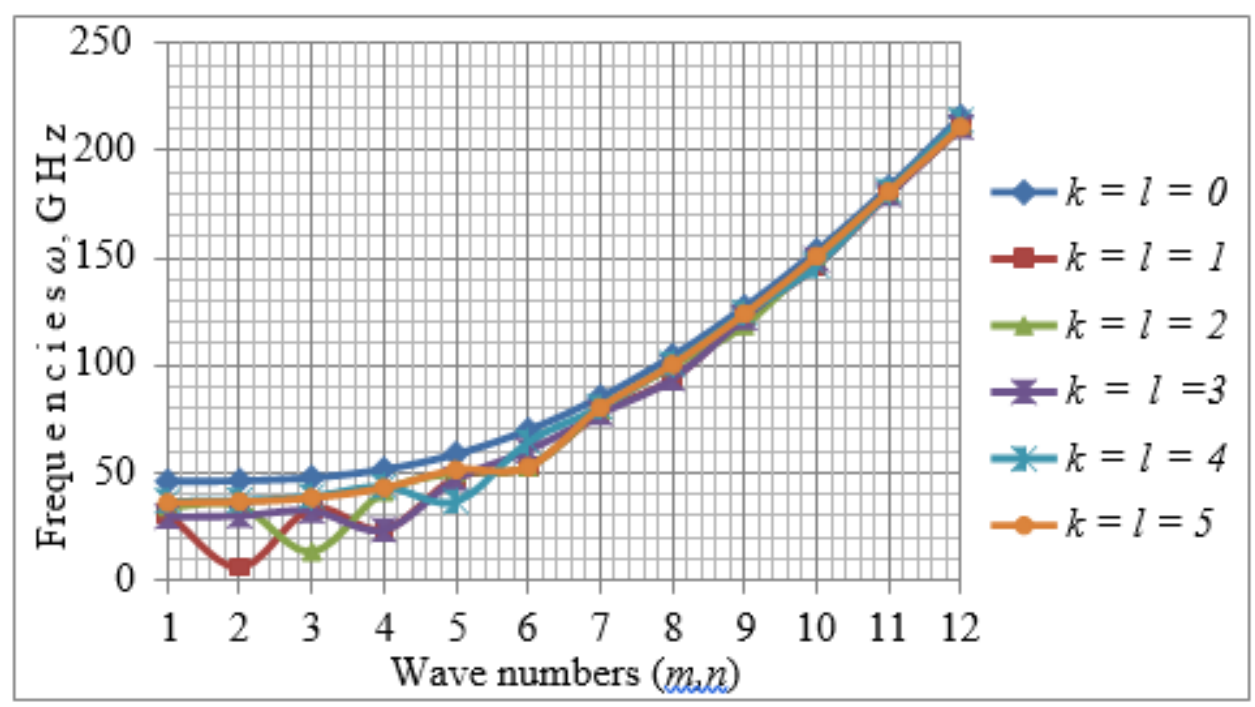

Figure 5. Frequency variation curves based on the wave numbers $(\mathrm{m}, \mathrm{n})$ for $12 \times 12 \mathrm{~m}$ shell 


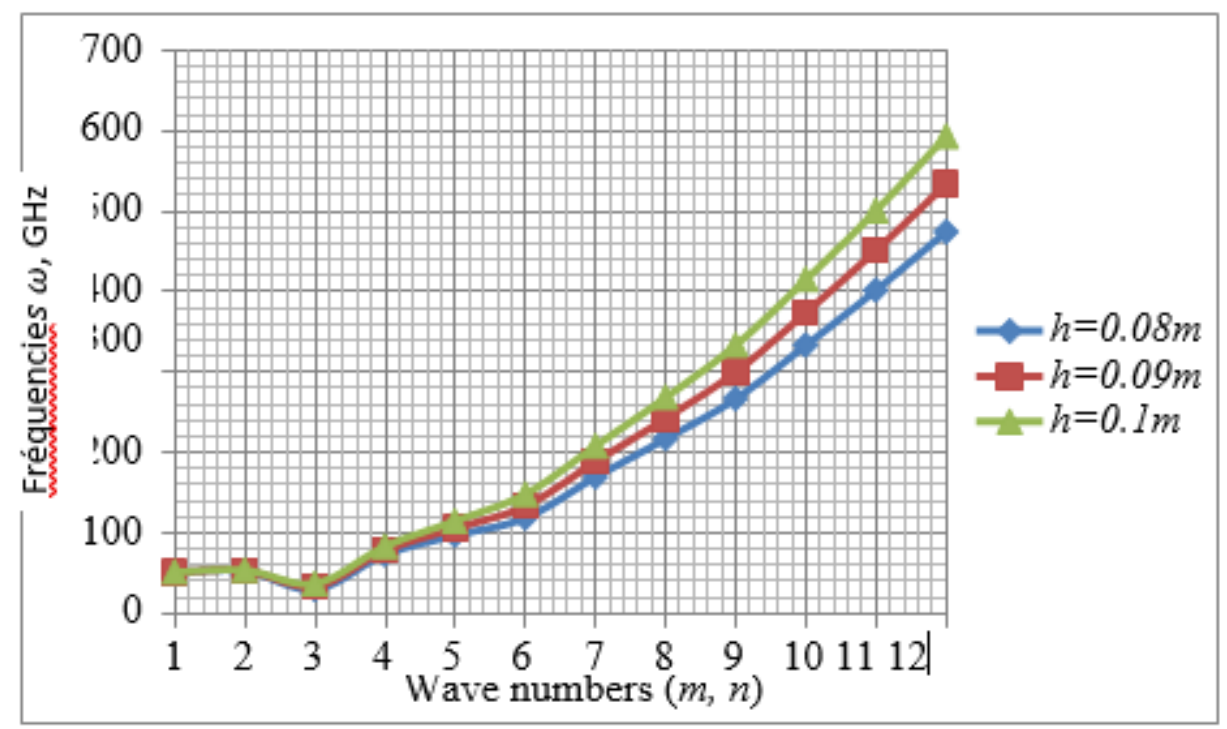

Figure 6. Frequency variation curves based on $8 \times 8 \mathrm{~m}$ shell thickness

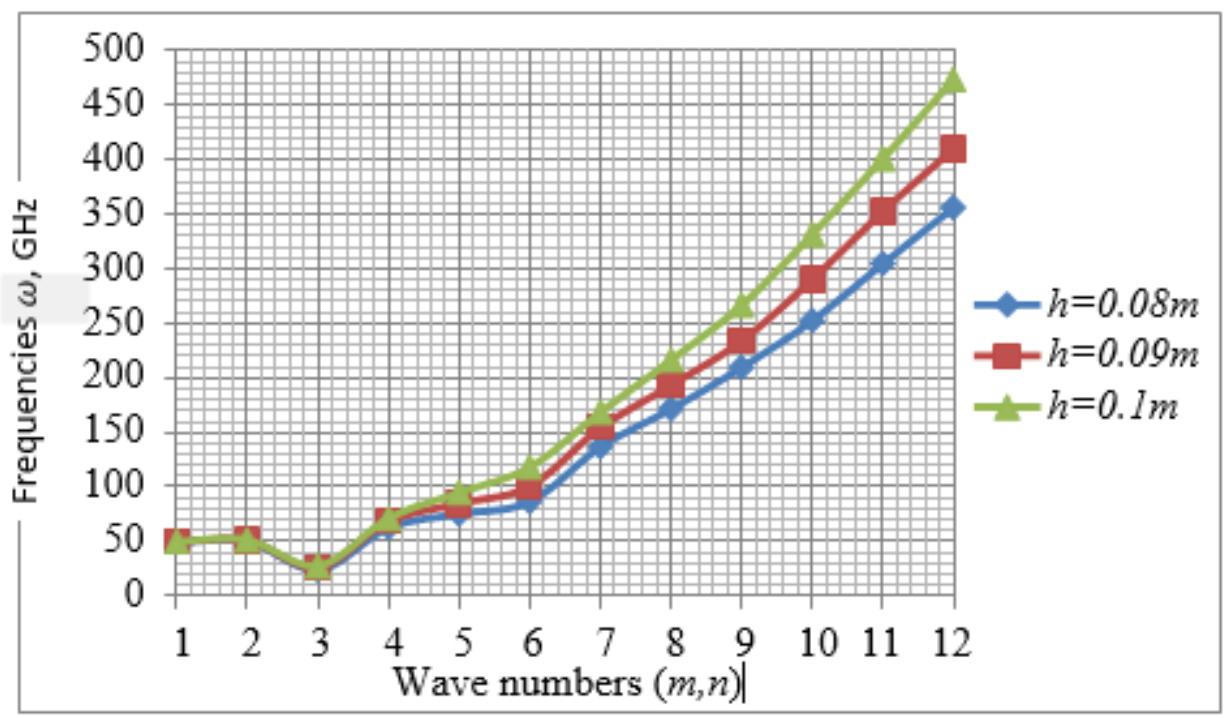

Figure 7. Frequency variation curves based on $12 \times 12 \mathrm{~m}$

shell thickness

Analysis of the curves of figures 4-7 shows that:

When the edge number $k=l$ is between 1 and 3, there is a minimum frequency for $m=k+1$ and $n=l+1$. As a result the dangerous sections will be the sections at the junctions from which occur rotations and shears of plane elements one in relation to the other.

When $k=l>3$ we have a minimum frequency for $m=1$ and $n=1$. In this case no shear in the central section.

There is a decrease in the minimum frequency for decrease in the edge number and thickness but there is decrease in the minimum frequency for increase in the shell dimension on the plane. Hence the need to provide damping devices in the structure. 


\section{Conclusion}

From this work we can retain the following:

1 - A numerical solving algorithm is proposed for the problem in free vibrations of low folded shells.

2 - The discret model of the used finite elements is described.

3 - Using Householder's QR transformations a numerical solving algorithm is developed for the eigenvalue problem of low folded shell.

4 - Mathematical models of low folded shells were proposed.

2 - Based on these geometric models the proposed method is validated for vibration analysis of low folded shells.

In perspective on the basis of the proposed model one can consider the development of a calculation method that takes into account the damping properties of the dissipative parts incorporated into the structure in order to reduce dynamic stress levels.

\section{References}

Ahmad, B. M. \& Zienkiewicz, O. C. (1970). Analysis of thick and thin shell structures by curved finite element. International Journal for Numerical Methods in Engineering, 2, 419-451. https://doi.org/10.1002/nme.1620020310

Arnoldi, W. E. (1951). The principle minimized iteration in the solution of the matrix eigenvalue problem. Quarterly of Applied Mathematics, 45, 17-29. https://doi.org/10.1090/qam/42792

Asokendu, S. \& Madhujit, M. (2004). Free vibration analysis of stiffned shells by the finite element technique. $\begin{array}{llll}\text { European Journal of } & \text { Mechanics-A/Solids, } & 23(1), & \text { 159-179. }\end{array}$ https://doi.org/10.1016/j.euromechsol.2003.11.001

Bate, K. J. (1982). Finite Element Procedures in Engineering Analysis, Prentice-Hall.

Brendel, B. \& Ramm, E. (1980). Linear and non-linear stability analysis of cylindrical shells. Computers and Structures, 12, 549-558. https://doi.org/10.1016/0045-7949(80)90130-3

Chen, X., Chen,H. L. \& Hu, x,L. (1999). Damping prediction of sandwich structures by order-reduction-iterative approach. Journal of Sound and Vibration, 222, 803-812. https://doi.org/10.1006/jsvi.1998.2131

Daya, E. M. \& Potier-Ferry, M. (2001). A numerical method for non-linear eigenvalue problems application to vibrations of viscoelastics structures. Computers \& Structures, 79, 533-541. https://doi.org/10.1016/S0045-7949(00)00151-6

El Damatty, A. A., Saafan, M. S. \& Sweedan, A. M. I. (2005). Dynamic characteristics of combined conical-cylindrical shells. Thin Walled Structures, 43, 1380-1397. https://doi.org/10.1016/j.tws.2005.04.002

Kim, K/ D., Liu, G. Z. \& Han, S. C. (2005). A resultant of I-nodesolid-sshell element for geometrically non-linearanalysis. Computational Mechanics, 35, 315-331. https://doi.org/10.1007/s00466-004-0606-9

Lanczos, C. (1950). An iteration method for the solution of the eigenvalue problem of linear differential and integral operators. Journal on Research of the National Bureau of Standards, 45, 255-282. https://doi.org/10.6028/jres.045.026

Leung, A. Y. T. (1995).Subspace iteration for complex symmetric eigenproblems. Journal of Sound and Vibration, 184, 627-637. https://doi.org/10.1006/jsvi.1995.0337

Nguyen-Thoi, T., Bui-uan, T., Liu, G. R. \& Vo-Duy, T. (2018). Static and free vibration analysis of stiffned flat shell by a cell-based smoothed discrete shear Gap method (CS-FEM-DSG3) using three-node triangular elements. International Journal of Computational Methods,15(6), 1850056. https://doi.org/10.1142/S0219876218500561

Patel, B. P., Ganapathi, M. \& Kamat, S. (2000). Free vibration characteristics of laminated composite joined conical-cylindrical shells. Journal of Sound and Vibration, 55, 920-930. https://doi.org/10.1006/jsvi.2000.3018

Pathak, R., Melani, M. \& Khane, R. (2019). Finite element modelling and free vibration analysis of RC shell and spatial structures for seismic evaluation. Proceeding of Recent Advances in Interdisciplinary Trends in Engineering and Applications (RAITEA). 
Parish, H. (1979). A critical survey of the 9-nodedegenerated shellelement with special emphasis on thin shell application and reduced integration. Computer Method in Applied Mechanics and Engineering, 20, 323-350. https://doi.org/10.1016/0045-7825(79)90007-0

Strang, G. (1980). Linear algebra and it s applications. New York Academic Press.

Wilkinson, J. H. (1988). The algebraic eigenvalue problem. Oxford University Press.

Zienkiewicz, O. C. (2000). The finite element method. Jordan.

\section{Copyrights}

Copyright for this article is retained by the author(s), with first publication rights granted to the journal.

This is an open-access article distributed under the terms and conditions of the Creative Commons Attribution license (http://creativecommons.org/licenses/by/3.0/). 